\title{
Les publications en physique au CNRS : combien, par qui et où ?
}

L'évolution rapide du paysage des publications scientifiques fait surgir beaucoup de questions et de discussions dans nos communautés. C'est une bonne chose, car cela nous concerne plus que jamais. Dans une démarche commune, I'Institut de Physique (INP) du CNRS et la Société Française de Physique (SFP) contribuent à ce débat en proposant des outils aux chercheurs, et en organisant des sessions d'informations (voir, par exemple, [1]).
Afin d'ancrer la discussion sur des bases plus solides, nous présentons ici une étude statistique sur les publications scientifiques dans le domaine de la physique en France, en particulier celles du CNRS. Les chiffres sont extraits directement de l'analyse du Web of Science ou de l'outil Zento du CNRS, qui recense la production scientifique par extraction de la base de données ISI Web of Science [http://wokinfo.com/] sur mot-clé prédéfini. Cette base de données est sans doute tronquée (comme toutes les bases de données), mais dans le domaine de la physique elle comporte 3500 revues, pour la plupart des journaux réguliers, et peu d'actes de colloque. Dans cet article, la « physique » correspond aux domaines de recherche tels que définis sur le Web of Science.

En 2013, un peu moins de 10000 articles de "physique " étaient publiés en France. $\mathrm{Si}$ nous nous intéressons aux données concernant toutes les unités affiliées au CNRS (UMR, UPR, UMS, UMI...), on repère ainsi environ $81 \%$ de toute la production scientifique de la France en physique. Les $19 \%$ restants concernent les articles signés par des unités sans mixité avec le CNRS, par d'autres EPST, EPIC (CEA, CNES, Onera...) ou des entreprises. On a constaté d'ailleurs que presque 16\% des auteurs français en physique renseignent leur affiliation de façon incorrecte et ne sont pas repérés par le mot clé "CNRS ". Sans doute la multiplication des tutelles incite les coauteurs à tronquer les signatures, mais une fraction non négligeable des physiciens-auteurs des unités du CNRS ne mentionnent même pas le nom de leur cotutelle, voire de leur propre employeur.

Au niveau mondial, et toutes thématiques confondues, presque 1,6 million d'articles paraissent par an. Plus de 130000 articles de physique, soit 8,6\% de l'ensemble, étaient publiés en 2013 dans le monde, un chiffre qui a augmenté d'un tiers depuis 2003. Presque 7500 articles en physique par an sont cosignés par une unité CNRS, soit 5,8\% de l'ensemble mondial en physique, ou $15 \%$ de la production totale du CNRS, estimée à 50000 par an. Au cours de la

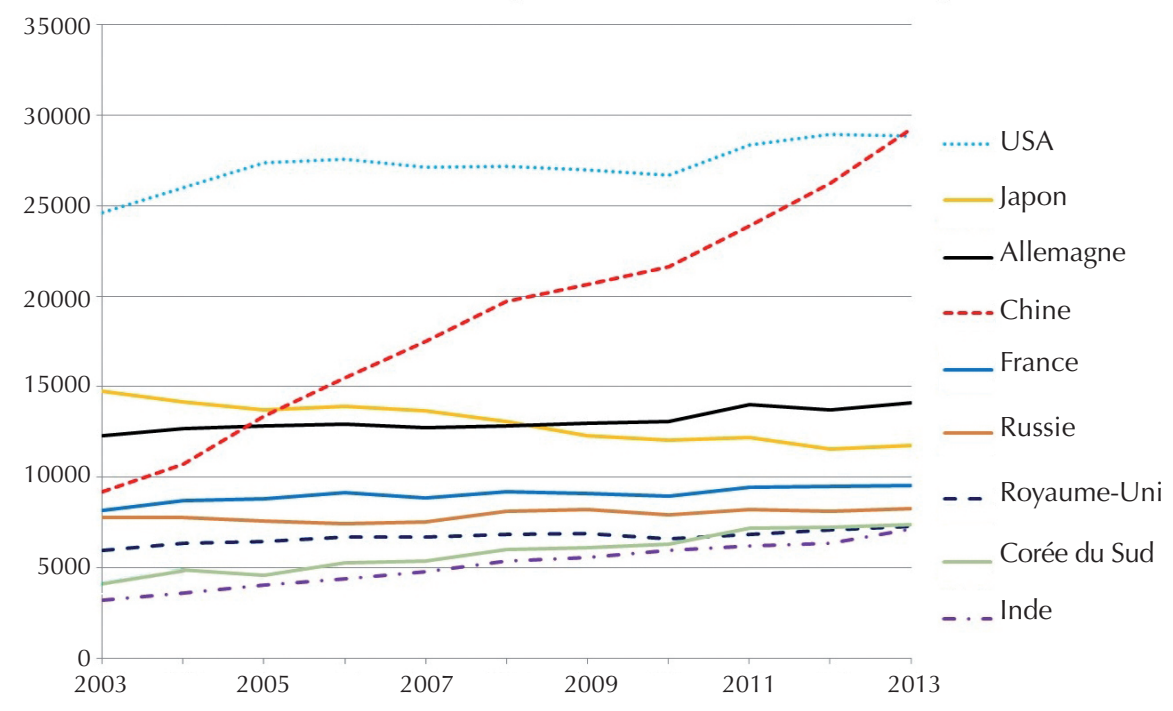

1. Évolution du nombre des publications en physique par pays pendant 10 ans. Entre 2003 et 2013, le nombre total est passé de 102000 à 137 000. (Données Web of Science) 


\section{Publications}

dernière décennie, le poids relatif des pays publieurs s'est modifié, comme le montre la figure 1, avec une forte croissance des publications venant de la Chine.

Si nous considérons «toute la physique au CNRS ", ceci comporte a priori la production des instituts INP et IN2P3 dans leur totalité, et une partie significative des publications des instituts INSIS et INSU. Par ailleurs, des publications peuvent être cosignées par des auteurs de différents instituts. Dans le cas d'auteurs de deux instituts différents, la publication compte, dans notre analyse, 0,5 pour chaque institut. Ce calcul « fractionné » est généralement utilisé pour pondérer les contributions collaboratives. De ce calcul résulte la structure fine de la répartition des publications, visible sur la figure 2. L'ensemble des instituts du CNRS (exception faite des sciences humaines et sociales) publie des articles en relation avec la physique. Si on compte directement les signataires d'un article, alors $50 \%$ de toutes les publications en physique au CNRS sont signées par au moins un auteur de l'INP. On peut par ailleurs observer (fig. 3) que presque deux tiers de la production scientifique de l'Institut de Physique du CNRS concerne vraiment « la physique »-définie dans le sens des domaines de recherche du Web of Science. Les figures 2 et 3 montrent que le périmètre de l'INP couvre une large palette de thématiques et que la physique est une activité intrinsèquement interdisciplinaire. Il y a une vraie synergie qui existe aux interfaces de la physique, et en particulier avec la chimie.

Au sein de la physique, on peut remarquer que la production scientifique mondiale est dominée en nombre d'articles par trois grandes thématiques : la physique appliquée, la physique de la matière condensée et la physique des matériaux. Cette répartition peut être retrouvée dans la distribution des principaux journaux dans lesquels les articles de l'INP apparaissent, avec Physical Review B (matière condensée) largement

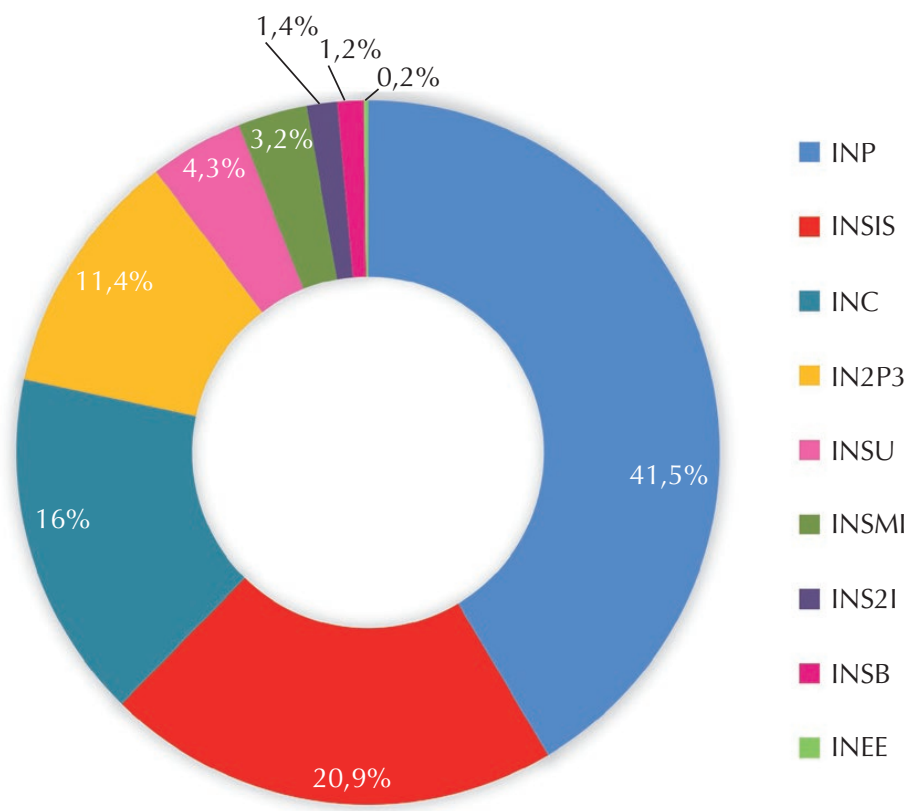

2. Répartition des publications en physique par institut du CNRS. Pour les publications signées par plusieurs (n) instituts, chaque institut obtient sa part fractionnée (1/n). (Données 2011-2012.)

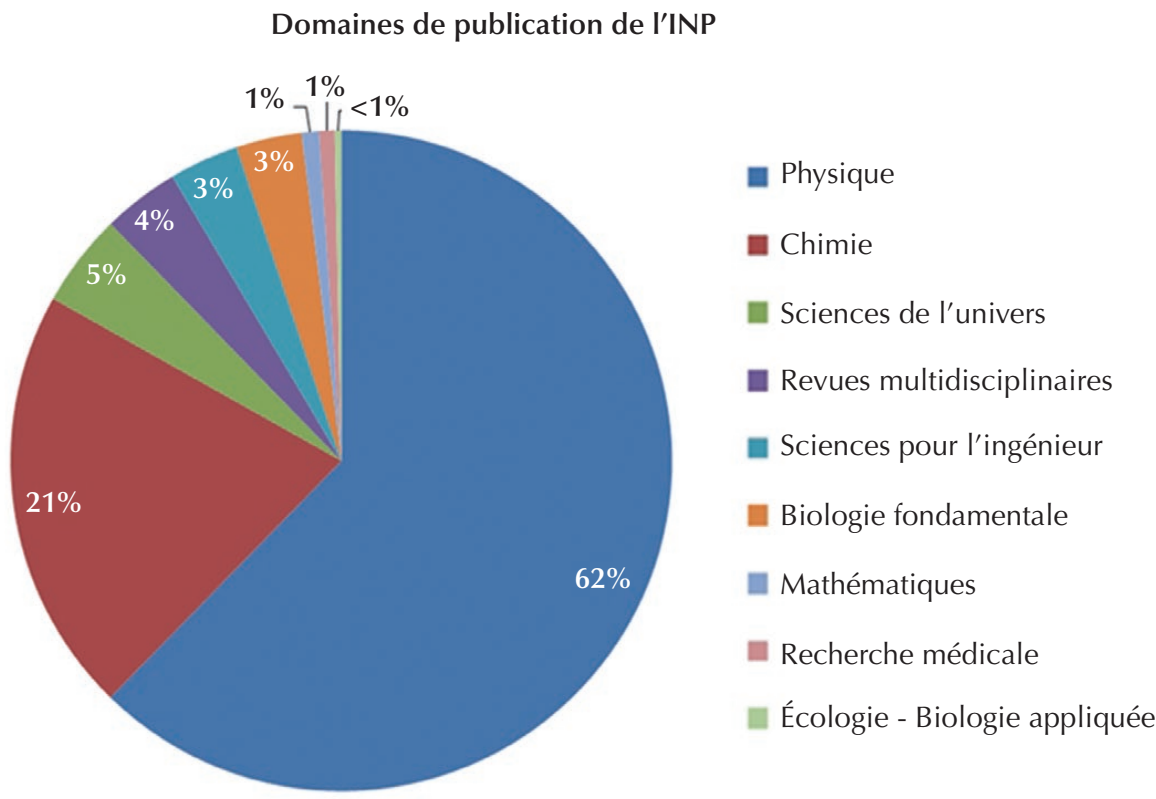

3. Répartition des catégories du Web of Science dans les publications de l'Institut de Physique du CNRS (un article peut se retrouver dans plusieurs catégories). (Données Zento 2013, utilisation de l'indexation OST, www.obs-0st.fr) 
en tête du classement. La figure 4 illustre (en rouge) le poids des articles écrits par des auteurs affiliés à l'INP sur la totalité des articles d'une revue. Les barres d'erreur de ce type de statistique sont évidemment grandes pour des journaux de petit volume. Il est remarquable de constater que $7 \%$ de toutes les publications dans les journaux de type "lettre blanche " (toutes thématiques confondues), comme Physical Review Letters (PRL) et EPL, sont signées ou cosignées par un auteur de l'Institut de Physique du CNRS !

Concluons sur le facteur d'impact (IF). Il est bien connu que le calcul d'un IF n'a de sens que pour l'ensemble des articles d'un journal, et que cette moyenne ne reflète jamais la qualité d'un article individuel [2]. Il va sans dire que, pour juger cette dernière, il n'y a pas de solution de calcul : il faut lire l'article ! Plus de $80 \%$ des journaux qui publient les articles de l'INP ont un IF $<4$, avec une valeur moyenne d'environ 2,8; un deuxième petit pic dans cette distribution peut être retrouvé autour de l'IF de Physical Review Letters (fig. 5). Le calcul de l'IF est malheureusement devenu un argument de "vente ", qui s'appuie sur une valeur moyennée sur une variété d'articles et de communautés trop différentes [2]. Même les grands journaux multidisciplinaires (tels que Nature ou Science) publient des articles à zéro citation!

Le graphique de la figure 5 montre bien que les publications de l'INP sont distribuées dans une large gamme de journaux de différents facteurs d'impact. La très grande majorité $(>80 \%)$ des articles sera citée au moins une fois au cours des cinq ans suivant leur parution, ce qui est très rassurant dans un domaine où certaines publications ne deviennent intéressantes qu'après plusieurs décennies. I

Martina Knoop (martina.knoop@univ-amu.fr), Lorenzo Brighigni et Bart van Tiggelen Institut de Physique du CNRS

\section{Références}

1• A. Henry et al., «Réflexions sur la "Voie dorée" des publications scientifiques", Reflets de la physique $\mathbf{3 0}$ (2012) 28-30.

2• « La SFP et l'INP du CNRS sensibilisent les physiciens à l'utilisation abusive des facteurs d'impact ", Reflets de la physique 32 (2013) 46.

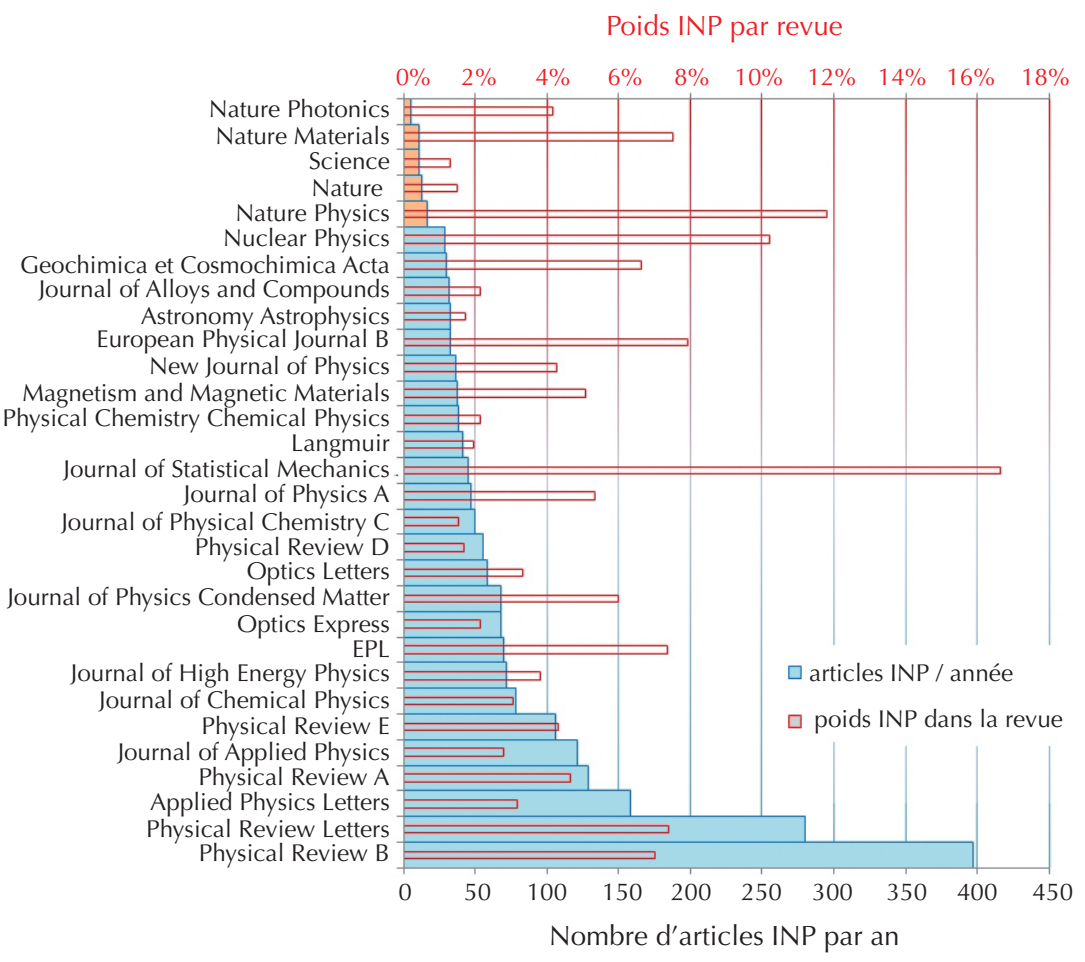

4. Nombre moyen annuel (entre 2007 et 2011) de publications signées par une unité rattachée à l'INP pour les 25 revues de physique dans lesquelles l'INP publie le plus (en bleu) et pour 5 revues " prestige » multidisciplinaires (en orange), et poids relatif (en rouge). (Données Web of Science 2007-2011.)

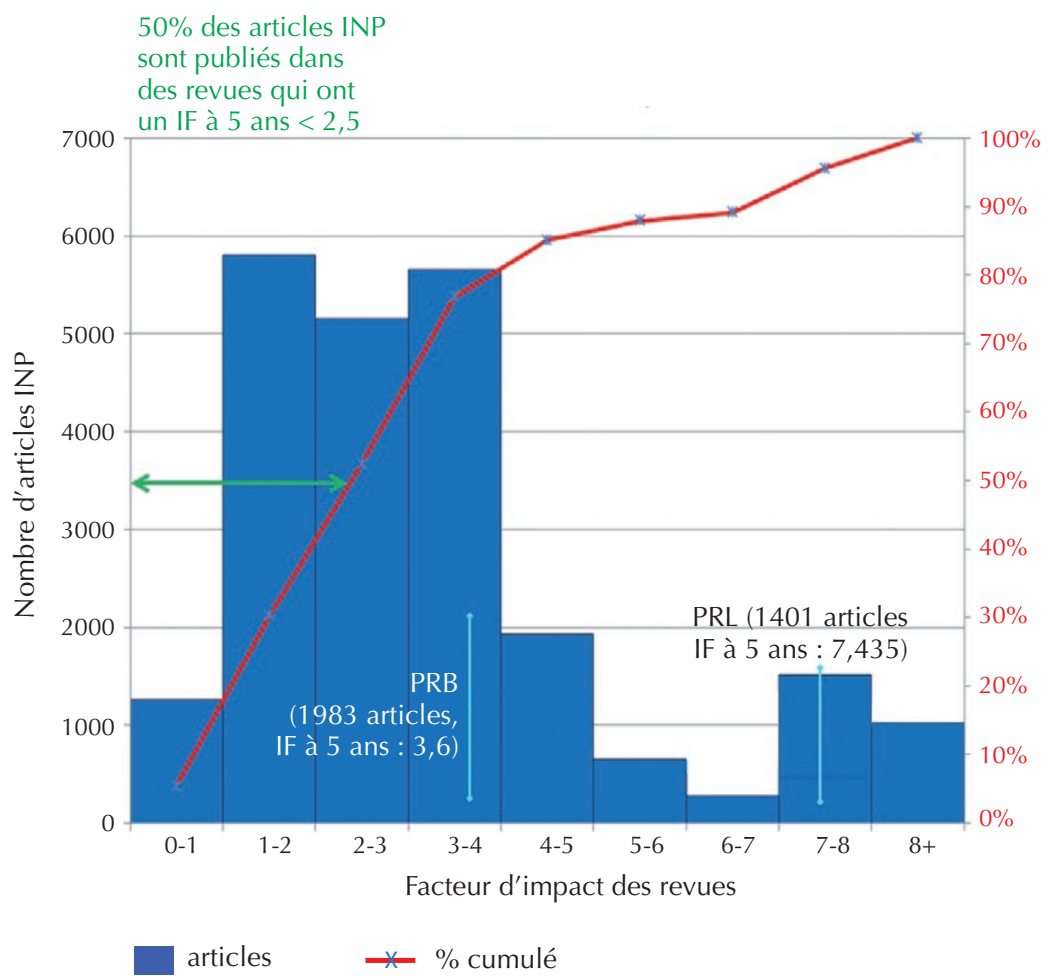

5. Nombre de publications de l'Institut de Physique du CNRS, en fonction du facteur d'impact de la revue. Ces chiffres sont cumulés sur 5 ans (2007-2011). La figure montre que 50\% des articles sont publiés dans des revues avec un facteur d'impact (IF) à 5 ans inférieur à 2,5. 